\title{
CONVERGENCE STUDIES FOR AN ADAPTIVE MESHLESS LEAST-SQUARES COLLOCATION METHOD
}

\author{
KA CHUN CHEUNG \& LEEVAN LING \\ Department of Mathematics, Hong Kong Baptist University.
}

\begin{abstract}
In this paper, we apply the recently proposed fast block-greedy algorithm to a convergent kernel-based collocation method. In particular, we discretize three-dimensional second-order elliptic differential equations by the meshless asymmetric collocation method with over-sampling. Approximated solutions are obtained by solving the resulting weighted least squares problem. Such formulation has been proven to have optimal convergence in $H^{2}$. Our aim is to investigate the convergence behaviour of some three dimensional test problems. We also study the low-rank solution by restricting the approximation in some smaller trial subspaces. A block-greedy algorithm, which costs at most $O\left(N K^{2}\right)$ to select $K$ columns (or trial centers) out of an $M \times N$ overdetermined matrix, is employed for such an adaptivity. Numerical simulations are provided to justify these reductions.

Keywords: ansa method, kernel-based collocation, adaptive greedy algorithm, elliptic equation
\end{abstract}

\section{INTRODUCTION}

Unsymmetric meshless kernel-based collocation methods, a.k.a. Kansa methods, have been used to solve various problems in science and engineering [1-5]. In 1990, Kansa solved time-dependent partial differential equation (PDEs) for the first time by such formulation using multiquadric $[6,7]$. Because of the ease of implementation and (potentially) high accuracy, the methods were widely adopted for over 10 years without any theoretical backup. In 2001, Hon and Schaback [8] showed that linear systems for Kansa methods could be singular. In order to obtain a non-singular linear system, the symmetric collocation method has been proposed [9], which requires a set of collocation-dependent basis functions. Until 2006, the convergence of the unsymmetric collocation method [10] has been proven provided that the collocation points are dense enough relative to the trial centers. The resulting linear system becomes over-determined, and it is natural to solve the resulting system in a least square sense. In [11], we investigate the convergence of the meshless collocation method for solving second-order elliptic equations in some bounded domain 1l. The theories showed that we have to impose certain weighting for the boundary part to obtain the stability estimate. Using reproducing kernels of the Sobolev space $H^{\mathrm{m}}(\Omega)$, we proved that the weighted least square solution converges to the analytical solution in an optimal rate $h^{\mathrm{m}-2}$ in $H^{2}$-norm. Numerical evidence in two dimensions also showed that the weighted least square problem gives more accurate results than the unweighted one [12].

So far, we did not address the problem of ill-conditioning, which depends on the choice of the kernel, its shape parameter, data points distribution, and literally everything in the partial differential equation. One solution is to look for a well-behaved subspace of the trial space so that the condition of the reduced linear system can be controlled. We proposed various subspace selection algorithms $[13,14]$ for such a purpose. The previously proposed sequential versions of adaptive greedy algorithms have a potentially high computational cost when the number of selections is large. Suppose we start from an $M \times N$ over-determined linear system with full rank $M$. If these sequential-greedy algorithms select $K$ columns out of the provided 
$N$, the cost would be $O\left(K^{4}+N K^{2}\right)$, i.e., it is $O\left(N^{4}\right)$ if $K \approx N$. Recently, a more efficient block-greedy algorithm [15] was proposed and the cost of selection is reduced to at most $O\left(N K^{2}\right)$, i.e., in the order as any direct method.

In this paper, we consider solving weighted least square problem by applying the blockgreedy algorithm for subspace selection. The two main benefits of employing adaptivity are: (1) to improve numerical stability by avoiding the problem ill-conditioning and (2) to improve efficiency by working with smaller linear systems. We aim to see how adaptivity would affect the theoretical convergence rate of the least-squares Kansa formulation.

\section{CONVERGENT WEIGHTED LEAST SQUARES KANSA FORMULATIONS}

In this section, we will discuss the weighted least square formulation and the block greedy algorithm in more detail. Let $\Omega \mathrm{C} \mathbb{R}^{d}$ be a bounded domain in $\mathbb{R}^{d}$. We strongly consider second-order elliptic differential equations subjected to a Dirichlet boundary condition on $\Gamma=\partial \Omega$ :

$$
\left\{\begin{array}{l}
L u=f \text { in } \Omega \\
u=g \text { in } \partial \Omega
\end{array}\right.
$$

where the differential operator with bounded coefficients is defined as

$$
\begin{aligned}
\mathrm{Lu}= & \sum_{i, j=1}^{d} \frac{\partial}{\partial x^{j}}\left(a^{i j}(x) \frac{\partial}{\partial x^{i}} u(x)\right)+\sum_{j=1}^{d} \frac{\partial}{\partial x^{j}}\left(b^{j}(x) u(x)\right) \\
& +\sum_{i=1}^{d} c^{i}(x) \frac{\partial}{\partial x^{i}} u(x)+d(x) u(x) .
\end{aligned}
$$

Let $\Phi$ be a symmetric positive definite kernel that reproduces a Sobolev space $H^{m}(\Omega)$ for some integer $m \geq 2+\left[\frac{1}{2}+(d+1)\right]$. Let $Z=\left\{z_{1}, \ldots, z_{n z}\right\}$ be a discrete set of trial centers in, $\Omega$, a.k.a. RBF centers. We define the finite dimensional trial space as

$$
u=u_{z, \Omega, \phi}:=\operatorname{span}\left\{\Phi\left(\cdot-z_{j}\right): z_{j} \in \mathrm{Z}\right\},
$$

within which we seek for a numerical approximation to the PDE. Let $X \subset \Omega$ and $Y \subset \Gamma$ be two discrete sets of collocation points in the domain $\Omega$ and on the boundary $\Gamma$. In order to measure the denseness of the data points $x \subset U \in\{\Omega, \Gamma\}$,we define the fill distance and separating distance as

$$
h z:=\sup _{\zeta \in U} \min _{z \in U}\|z-\zeta\| l_{2}\left(\mathbb{R}^{d}\right) \text { and } q z:=\frac{1}{2} \min _{\substack{z_{i}, z_{j} \in U \\ z_{i} \neq z_{j}}}\left\|z_{i}-z_{j}\right\| l_{2}\left(\mathbb{R}^{d}\right),
$$

respectively, and the mesh ratio is given by $\rho_{x}:=h_{x} / q_{x}$.

Assumption 2.1 We assume that he bounded domain $\Omega$ has a piecewise $C^{m}$-boundary $\Gamma$ so that $\Omega$ is Lipschitz continuous and satisfies an interior cone condition. Also, the functions fand $g$ in (1) are smooth enough to admit a classical solution $u^{*} \in H^{m}(\Omega)$. The operator $L$ as in (2) is a strongly elliptic operator with coefficients belonging to $W_{\infty}^{m}(\Omega)$. Let $\Phi$ be a translation-invariant symmetric positive definite kernel that reproduces kernel of $H^{m}(\Omega)$ for 
some integer $m \geq 2+\left[\frac{1}{2}(d+1)\right]$. Let the trial space be defined as in (3) by a discrete set oí trial centers $Z$. Let $X=\left\{x_{1}, \ldots, x_{n x}\right\}$ be another discrete set of PDE collocation points in $\Omega$ and $Y=\left\{y_{1}, \ldots, y_{n Y}\right\}$ be a set of boundary collocation points on $\Gamma$. We assume the set $Z$ of discrete trial centers to be sufficiently dense with respect to $\Omega, \Phi$, and $L$ but independent of the solution, and these three sets of points $X, Y$ and $Z$ to be asymptotically quasi-uniform. That is, there exist constants $\Upsilon_{\mathrm{X}}>1$ such that

$$
\Upsilon_{x}^{-1} q_{x} \leq h_{x} \leq \Upsilon_{x} q_{x} \cdot \text { for } x \in\{X, Y, Z\} .
$$

Under the above standard smoothness assumptions for high-order convergence, the following theorem ensures the convergence of a class of weighted least square (WLS) formulations. Most importantly, the necessary ratio of over-sampling in the over-determined Kansa formulation is linear. It also specifies the scaling factors required for boundary collocations, i.e., the $h_{X}$ and $h_{Y}$ dependent term in front of the discrete $Y$ norm in (5).

Theorem 2.2 (Theorem 2.8 [11]) Suppose Assumption 2.1 holds, see details in [11]. Let $u_{X, Y, Z}^{\theta}$ be the weighted least-squares solution defined as

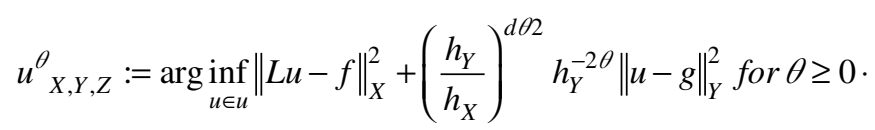

Moreover, the relative mesh norm $h_{x} / h_{\mathrm{z} \cup \mathrm{y}}$ and $h_{z} / h_{\mathrm{z} \cup \mathrm{y}}$ are sufficiently small and satisfy the denseness requirement

$$
C_{\Omega, L, m}\left(h_{X}^{m-2}+h_{Y}^{m-2}\right) h_{Z}^{-m+2} p_{Z}^{m-1}<1 / 2,
$$

for some constant $C_{\Omega, L, m}$ that does not depend on the sets of points $\mathrm{X}, Y$, and $Z$. Then for any $0 \leq \theta \leq 2$, the error estimates

$$
\left\|u_{X, Y, Z}^{\theta}-u^{*}\right\| H^{2}(\Omega) \leq C\left(1+h_{X}^{\frac{(\theta-2) d}{4}} \frac{(\theta-2)(d-4)}{h_{Y}}+h_{Y}^{-3 / 2} h_{Z}^{2}\right) h_{Z}^{m-d / 2-2}\left\|u^{*}\right\| H^{m}(\Omega)
$$

and

$$
\left\|u_{X, Y, Z}^{\theta}-u^{*}\right\| H^{2}(\Omega) \leq C\left(1+h_{X}^{\frac{(\theta-2) d}{4}} h_{Y}^{\frac{(\theta-2)(d-4)}{4}}+h_{Y}^{-3 / 2} h_{Z}^{2}\right) h_{Z}^{m-d / 2-2}\left\|u^{*}\right\| H^{m}(\Omega)
$$

For $\geq 2+\left[\frac{d+1}{2}\right]$ and $m>3+d / 2$, respectively, hold for some constant $C$ that depends only on $\Omega, \Phi, L$ and uniformity constants $\Upsilon_{X}, \Upsilon_{Y}$ and $\Upsilon_{Z}$ in (4).

Denoting the number of points in $\mathrm{X}, \mathrm{Y}$ and $\mathrm{Z}$ be $n_{X}, \mathrm{n}_{Y}$ and $n_{Z}$, respectively. The matrix form of the least square problem must be an over-determined system since the number of collocation points $X$ and $Y$ must be larger than the number of trial centers so as to satisfy the denseness requirement suggested in Theorem 2.2. Suppose the above collocation method yields an $\left(n_{X}+n_{Y}\right) \times n_{Z}$ matrix system $\mathrm{A} \lambda=b$ for identifying the unknown coefficient vector $\mathrm{A} \in \mathbb{R}^{n z}$ and, hence, specifying the numerical solution 


$$
u_{X, Y, Z}^{\theta}=\sum_{\zeta_{j} \in Z} \lambda_{j} \Phi\left(\left\|\cdot-\zeta_{j}\right\|\right) .
$$

We employ the block-greedy algorithm in [15] deal with such problems. For the algorithm to run, the original matrix $A=A((X, Y), Z)$ does not need to be pre-computed.

Instead, only some of its components, which are determined based on the selected subset of collocation points $(X, Y)_{\mathrm{k}}$ and trial centers $Z_{\mathrm{k}}$, are required for the algorithm to run. In each step, only the solutions of subsystems

$$
A\left((X, Y)_{k}, Z\right) \lambda_{k}=b_{k} \text { and } A\left((X, Y)_{k}, Z_{k}\right)^{T} v k=\lambda k
$$

are required to compute the primal and dual residuals. These sub-systems can be efficiently solved by carefully updating the QR factorizations of the expanding sub-matrices. To improve stability and robustness, the algorithm uses more selected collocation points than trial centers in all sub-systems and tries to double the numbers of points in each step. An iterative stopping criterion is implemented to select a subset of $K$ trial basis so that the condition number of $\mathrm{A}\left((\mathrm{X}, \mathrm{Y})_{\mathrm{k},} \mathrm{Z}_{\mathrm{K}}\right)$ is below the user-specified tolerance. The result of applying the block-greedy algorithm is a subset of $K$ trial centers out of the input $n_{\mathrm{Z}}$. The coefficients of the adaptive least-squares Kansa solution is then given by the least-squares solution to $\mathrm{A}\left((\mathrm{X}, \mathrm{Y}), \mathrm{Z}_{\mathrm{K}}\right) \lambda_{\mathrm{K}}=b$, in which we use all available collocation conditions. The coefficients associated with the selected $\mathrm{K}$ trial basis are specified by $\lambda K \in \mathbb{R}^{K}$ and those for the unselected are set to be zero. As a demonstration, Figure 1 shows the error profiles when solving a modified Helmholtz problem with Neumann boundary by a Kansa method with the (unscaled) GA trial function. To set up the method, it is not trivial tell the optimal number of trial basis required a priori and is not straightforward to uniformly distribute data sites in the Dupin cyclide. With "adaptivity" in place, a proper set of trial basis will be selected to yield better accuracy in a smaller amount of time. We refer readers to the original article for the details of the block-greedy algorithm. In the next section, we will examine

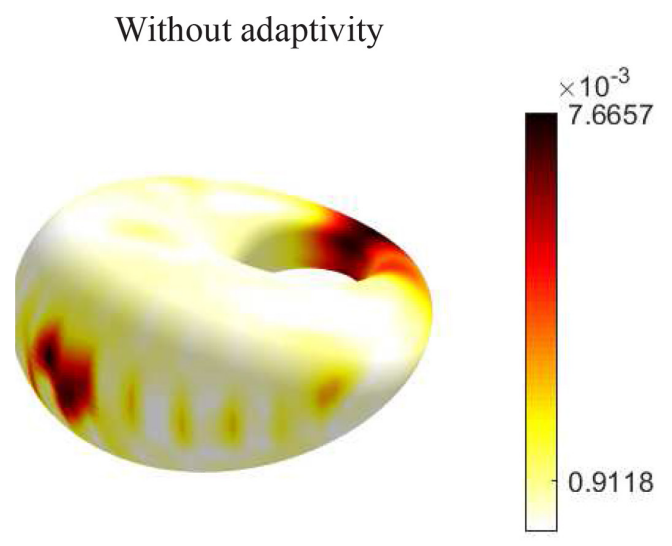

By block-greedy algorithm

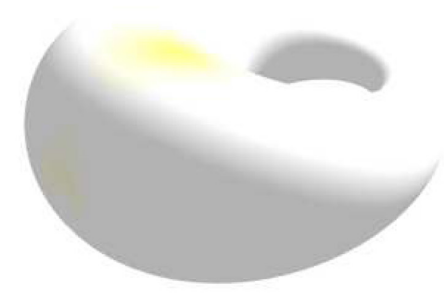

Using 3613 out of 9365 trial functions

Figure 1: Numerical error of Kansa solutions (left) using all the trial functions and (right) using a subset selected by the block-greedy algorithm. 
the convergence behaviour when we apply the adaptive algorithm to the convergent formulation in (5).

\section{NUMERICAL DEMONSTRATION}

In this section, we present two numerical experiments to illustrate the effects of add-on adaptivity to the efficiency and accuracy of the weighted least-squares Kansa formulation. We use the Whittle-Matérn-Sobolev kernel given as:

$$
\Phi(x):=\|\varepsilon x\|_{2}^{m-d / 2} \kappa_{m-d / 2}\left(\|\varepsilon x\|_{2}\right) \text { for all } x \in \mathbb{R}^{d},
$$

where $\kappa_{v}$ is the Bessel function of the second kind. The parameters m controls the smoothness and $\epsilon$ controls the shape of the kernel. We know that $\Phi$ is a reproducing kernel for $H^{m}\left(\mathbb{R}^{d}\right)$ for any $m>\mathrm{d} / 2$.

All numerical simulations are implemented on a workstation equipped with Ten cores Xeon E5-2690 v2 3.0 GHz CPUs, 128GB RAM running CentOS Linux version 6. The MATLAB code for the block-greedy algorithm can also be downloaded from [16].

\subsection{Example 1}

In this example, we consider solving a second-order elliptic equation on a rectangular domain $\Omega=[-1,1]^{3}$ in $\mathbb{R}^{3}$. i.e.,

$$
\left\{\begin{aligned}
-\Delta u+(1+x) u & =f \text { in } \Omega \\
u & =g \text { on } \partial \Omega .
\end{aligned}\right.
$$

The analytical solution is set to $u(x, y, z)=\sin (\pi x / 2) \cos (\pi y / 2) \cos (\pi z / 2)$ and the right-hand functions $f$ and $g$ are computed analytically. The parameters are set to $\varepsilon=0.7$ and $m=6$ being the smoothness of the kernel. These values are chosen to yield more noticeable demonstrations. In practice, one can simply use any parameters with the block-greedy subspace selection algorithm. In Figure 2, we show the distribution of the regular trial centers and some selected trial centers. The original $n_{Z}=4,913$ trial centers are evenly distributed in $\Omega$. Only $K=1,827$ trial centers are selected out of these $n_{Z}$ trial centers. It is obvious to see that the selection are rather uniform over both interior and the boundary of $l$, also see Figure 3 .

In the process of block-greedy algorithm, one measure for the performance of point selection is the condition number of the reduced matrix. In all (double precision) computations,
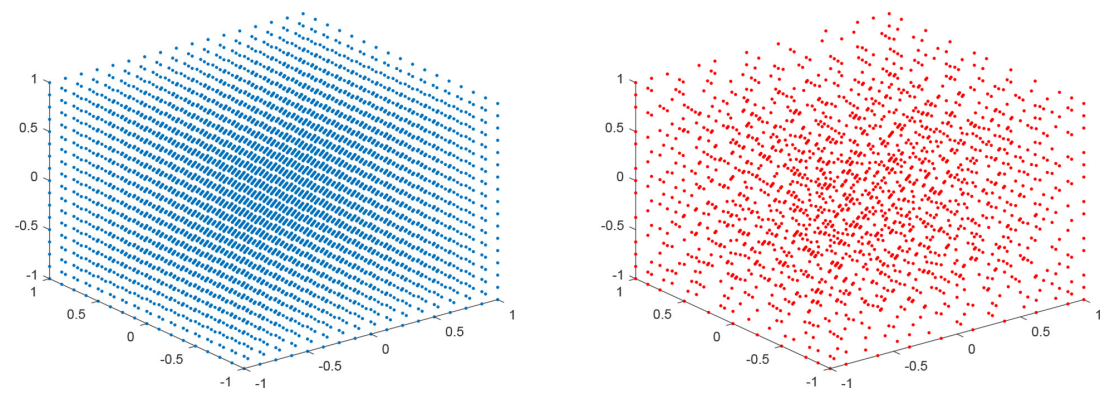

Figure 2: Example 1: A schematic demonstration of (left) all $n_{z}=4,913$ trial centers and (right) the subset of $K=1,827$ selected by the block-greedy algorithm. 


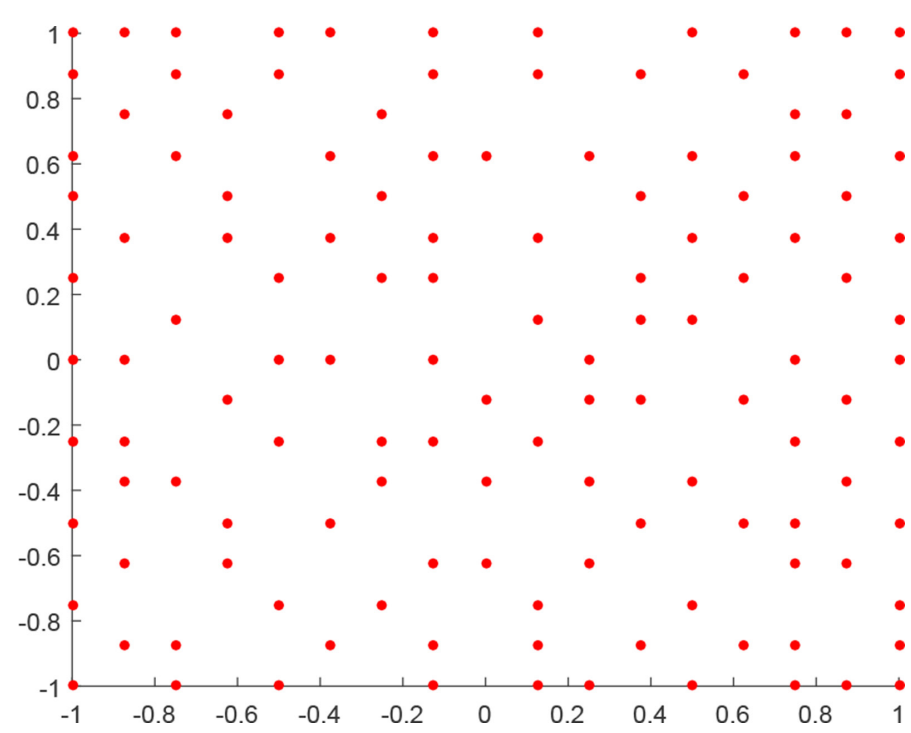

Figure 3: Example 1: The selected trial centers on the top layer $z=1$.
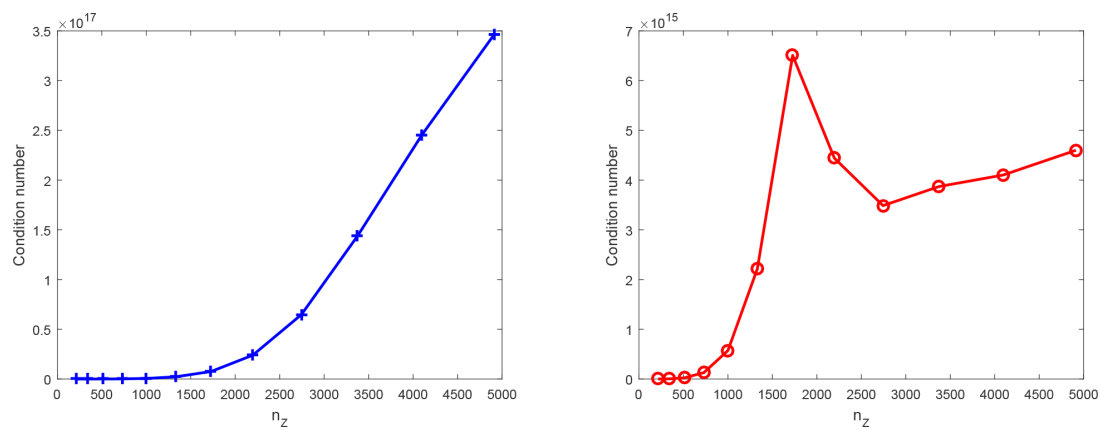

Figure 4: Example 1: The condition numbers of (left) the original $\left(n_{\mathrm{X}}+n_{\mathrm{Y}}\right) \times n_{\mathrm{Z}}$ matrix without any selection and (right) of the reduced matrices using only the selected trial basis.

we use a tolerance of $10^{16}$ in the "condition number" stopping criterion in the block-greedy algorithm. Without any surprise, the condition numbers of the resulting reduced matrices are at most $10^{16}$. In Figure 4, we show the condition number of the full over-determined system of the WLS without adaptivity and those of the reduced system. We can see that the condition numbers of the original system increase as the numbers of trial centers increase. When we use all $n_{z}=4,913$ trial centers, the condition number becomes $10^{17}$. However, with the blockgreedy algorithm in place, the condition numbers of the reduced systems remain smaller than the prescribed tolerance. This is the direct consequences of the subspace selection by design.

One of the main reasons of using subspace selection is that it can select an appropriate subspace from the given trial space (determined by all the other user's settings) so that the obtained approximation with the subspace yields a similar performance. Using the subspace to do the approximation can also reduce the problem size, and hence the computational time. 
Figure 5 shows both the computational times and the numbers of selected trial basis with respect to different numbers of total basis $n_{\mathrm{Z}}$. When the number of trial centers is small, say $n_{\mathrm{Z}} \leq 2,000$, the over-determined systems do not suffer the problem of ill-conditioning, and it is unnecessary to make the selection. Yet, employing the block-greedy algorithm does not increase the computational time significantly. When the numbers of selected trial basis stagnate (due to ill-conditioning), we can see that the computational times of the adaptive approach grow slowly, i.e., $O\left(n_{\mathrm{z}}\right)$. We can also see from Figure 5 that the numbers of selected points keep at around 1,800 when the numbers of trial centers are greater than 2,000. These are the situations when the user's setups yield ill-conditioned linear systems and are exactly when the subspace selection becomes significant. Finally, we show the $H^{2}$-error with respect to the mesh norm $h_{Z}$ in Figure 6 . The $H^{2}$-error for the adaptive-WLS solution with block-greedy algorithm is no worse (or slightly better) than that the one from the full
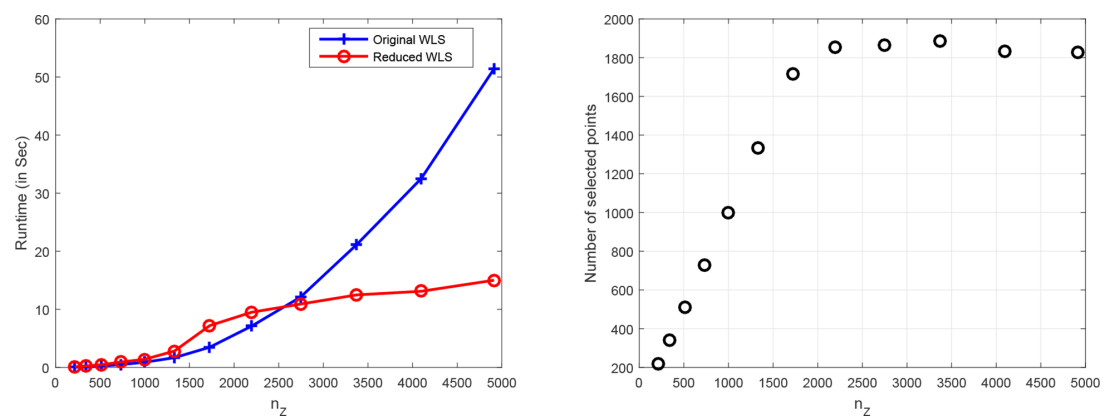

Figure 5: Example 1: (left) computational time for obtaining the convergent weighted least square solution with and without subspace selection, and (right) number of selected trial centers by the block-greedyalgorithm.

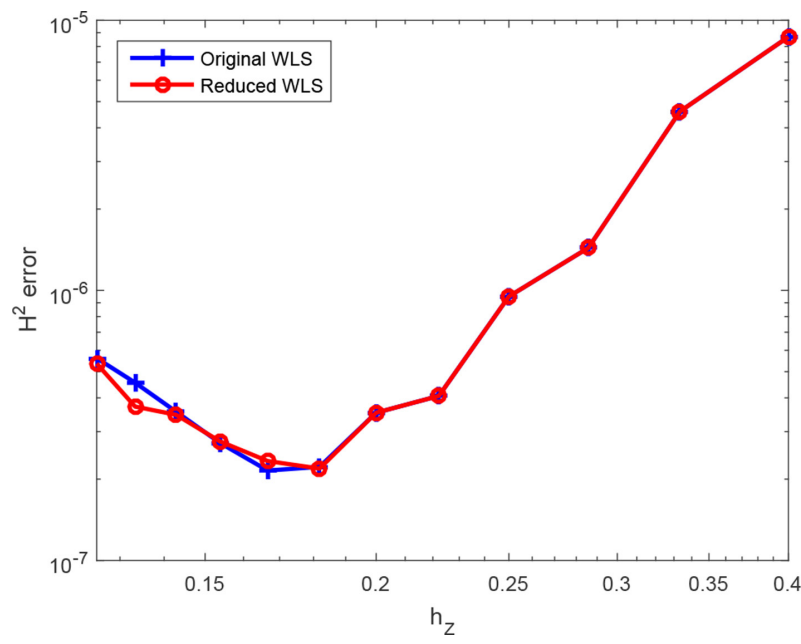

Figure 6: Example 1: The $\mathrm{H}^{2}$-convergence profile for the convergent weighted least square solution with and without adaptivity. 
system. Seeing no loss in accuracy is a good news. By using the block-greedy algorithm, we gain efficiency by significantly reducing a large number of trial centers to a smaller subset.

\subsection{Example 2}

In this example, we investigate the convergence behaviour for the different smoothness of the kernel. In this case, we consider solving the modified Helmholtz equation with wave number one whose analytical solution is set to

$$
u(x, y, z)=e^{-x^{2}-2 y^{2}-z^{2}}\left(x^{2}+y^{2}\right) .
$$

When the smoothness of kernel is $m$, it means that the corresponding reproducing kernel Hilbert space, a.k.a. the native space, is norm-equivalent to $H^{m}(\Omega)$. In our simulations, we study the convergence behaviour for $m=5,6,7$. We can see from Figure 7 that the numerical convergence rates are nearly 4.43, 6.17 and 8.63 , respectively, before the problem of ill-conditioning kicks in. In all tested $m$, the rates are all faster than $\mathrm{m}-2$ predicted by theories.
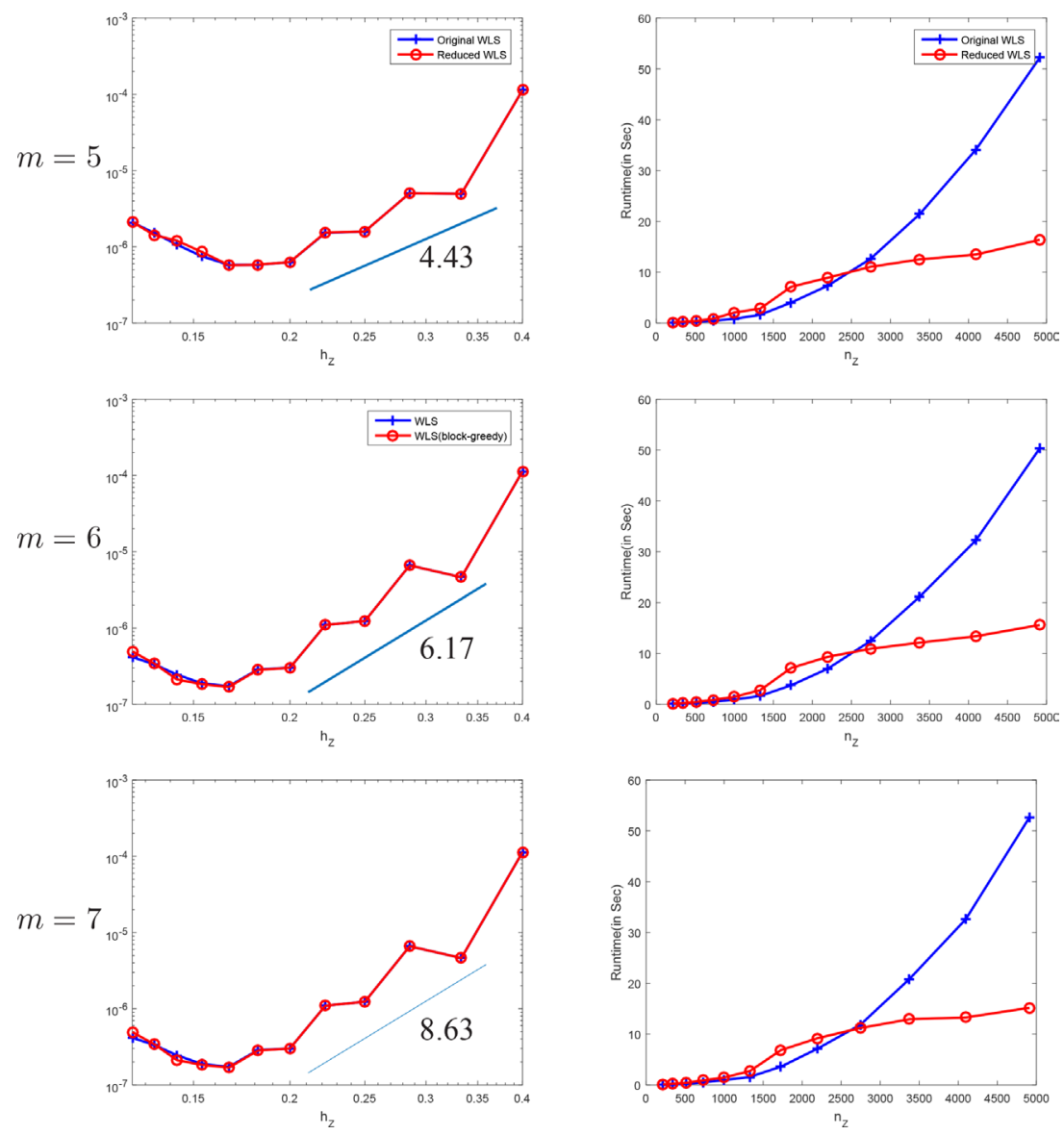

Figure 7: Example 2: The $H^{2}$-error profiles and computational times for various kernel with smoothness $m$. 
Similar to Example 1, we see that the $H^{2}$-errors are similar with or without the block-greedy algorithm. However, the computational time can be reduced significantly. As an example, the algorithm selects $K=1,839$ trial basis out of the 4,913 total, the computational time for solving the resultant least-squares system becomes one-fifth of the original one.

\section{CONCLUSION}

In this paper, we consider using an adaptive-weighted least squares Kansa formulation to solve second-order elliptic differential equation. In our previous work, we showed that such weighted least square solution is convergent in $H^{2}$ optimally and the order of convergence is related to the smoothness of the kernel. The proven convergence results give theoretical support to the method and the proper way to set up Kansa method. Yet, they do not solve the problem of ill-conditioning commonly seen in meshless methods with global kernels. In order to tackle this problem, we apply a newly proposed block-greedy algorithm for subspace selection. The fast block-greedy algorithm is an improvement of the sequential-greedy algorithm. The block-greedy takes only $O\left(N K^{2}\right)$ when the sequential-greedy takes $O\left(K^{4}+N K^{2}\right)$ when we want to select $\mathrm{K}$ trial centers out of $\mathrm{N}$ trial centers. This extra adaptivity can reduce computational costs. The remaining question is its effect on the accuracy. Numerical demonstrations show that the accuracies of lower-rank solutions obtained from smaller trial subspaces are as good as those from the whole space.

\section{REFERENCES}

[1] Fürst, J. \& Sonar, T., On meshless collocation approximations of conservation laws: preliminary investigations on positive schemes and dissipation models. ZAMMZeitschrift fïr Angewandte Mathematik und Mechanik Journal of Applied Mathematics and Mechanics, 81(6), pp. 403-415, 2001.

[2] Zhou, X., Hon, Y. \& Cheung, K., A grid-free, nonlinear shallow-water model with moving boundary. Engineering Analysis with Boundary Elements, 28(9), pp. 1135-1147, 2004.

http://dx.doi.org/10.1016/s0955-7997(03)00124-3

[3] Kansa, E.J. \& Geiser, J., Numerical solution to time-dependent 4D inviscid Burgers' equations. Engineering Analysis with Boundary Elements, 37(3), pp. 637-645, 2013. http://dx.doi.org/10.1016/j.enganabound.2013.01.003

[4] Li, W., Li, M., Chen, C.S. \& Liu, X., Compactly supported radial basis functions for solving certain high order partial differential equations in 3D. Engineering Analysis with Boundary Elements, 55(SI), pp. 2-9, 2015.

[5] Pang, G., Chen, W. \& Fu, Z., Space-fractional advection-dispersion equations by the Kansa method. Journal of Computational Physics, 293(SI), pp. 280-296, 2015.

[6] Kansa, E.J., Multiquadricsa scattered data approximation scheme with applications to computational fluid-dynamics-I surface approximations and partial derivative estimates. Computers \& Mathematics with applications, 19(8), pp. 127-145,1990. http://dx.doi.org/10.1016/0898-1221(90)90270-T

[7] Kansa, E.J., Multiquadrics-a scattered data approximation scheme with applications to computational fluid-dynamics-II solutions to parabolic, hyperbolic and elliptic partial differential equations. Computers \& Mathematics with Applications, 19(8-9), pp. 147-161, 1990. http://dx.doi.org/10.1016/0898-1221(90)90271-k 
[8] Hon, Y. \& Schaback, R., On unsymmetric collocation by radial basis functions. Applied Mathematics and Computation, 119(2), pp. 177-186, 2001.

[9] Fasshauer, G.E., Solving differential equations with radial basis functions: multilevel methods and smoothing. Advances in Computational Mathematics, 11(2-3), pp. 139-159,1999.

[10] Schaback, R., Convergence of unsymmetric kernel-based meshless collocation methods. SIAM Journal on Numerical Analysis, 45(1), pp. 333-351, 2007.

[11] Cheung, K.C., Ling, L. \& Schaback, R., $\mathrm{H}^{2}$-convergence of least squares kernel collocation method, Submitted 2016.

[12] Hu, H., Chen, J. \& Hu, W., Weighted radial basis collocation method for boundary value problems. International Journal for Numerical Methods in Engineering, 69(13), pp. 2736-2757, 2007.

[13] Ling, L. \& Schaback, R., Stable and convergent unsymmetric meshless collocation methods. SIAM Journal on Numerical Analysis, 46(3), pp. 1097-1115, 2008.

[14] Ling, L. \& Schaback, R., An improved subspace selection algorithm for meshless collocation methods. International Journal for Numerical Methods in Engineering, 80(13), pp. 1623-1639, 2009.

[15] Ling, L., A fast block-greedy algorithm for quasi-optimal meshless trial sub-space selection. SIAM Journal on Scientific Computing, 38(2), pp. A1224-A1250, 2016.

[16] Ling, L., available at: http://www.math.hkbu.edu.hk/ lling/blockgreedy.m. 